\title{
A STUDY OF INTERFACE DERMATITIS WITH CLINICAL CORRELATION
}

\author{
Suja Ajoy Kumar' ${ }^{1}$ G. Nandakumar²
}

\section{HOW TO CITE THIS ARTICLE:}

Suja Ajoy Kumar, G. Nandakumar. "A Study of Interface Dermatitis with Clinical Correlation". Journal of Evolution of Medical and Dental Sciences 2015; Vol. 4, Issue 42, May 25; Page: 7343-7351,

DOI: $10.14260 /$ jemds/2015/1066

ABSTRACT: BACKGROUND: Interface dermatitis (ID) is defined as an inflammatory skin disease in which the junction between the papillary dermis and the epidermis is obscured. They are so named because they are cell mediated immune reactions whose targets are basal keratinocytes that reside above the dermo-epidermal junction. Interface dermatitis encompasses multiple clinical entities with diverse histological features. This study is aimed at understanding the various associated histological features with clinical correlation of conditions in which Interface Dermatitis is the most important histopathologic finding. This will help us in arriving at a more specific diagnosis by light microscopy. MATERIALS AND METHODS: The study was conducted over a period of two and a half years. Skin biopsies showing interface dermatitis as a major histological finding were studied in detail and classified according to Le Boit's classification. PAS staining was done to highlight pathology of basement membrane. Normal skin biopsies \& biopsies with an unrelated condition were taken as control. Correlation between clinical features, clinical diagnosis and histological diagnosis was assessed. RESULTS: 71 cases showing interface dermatitis were studied. The most common age range was between 50-59 years with a female preponderance. Majority of the cases belonged to type I interface dermatitis with lichen planus being representative of this type. PAS staining revealed basement membrane disruption in many of the cases. CONCLUSION: Interface dermatitis includes diverse entities which have overlapping features at the clinical and histopathological level. Hence, a detailed light microscopic examination aided further by special tests such as immunofluorescence will help at arriving at a definitive diagnosis. More studies are also needed in order to identify specific features to redefine each of the entities.

KEYWORDS: Interface Dermatitis, Skin, Le Boit's classification.

INTRODUCTION: One of the most challenging aspects in dermatopathology is to try to make specific diagnosis of inflammatory skin diseases. Criteria now exist for the microscopic diagnosis of many inflammatory skin diseases and this has increased the utility of biopsy procedures in inflammatory conditions. ${ }^{1}$

Interface dermatitis is defined as an inflammatory skin disease in which the junction between the papillary dermis and the epidermis is obscured. They are so named because they are cell mediated immune reactions whose targets are basal keratinocytes that reside above the dermoepidermal junction. ${ }^{1}$ The lymphocytes infiltrate the basal layer of the epidermis and cause cytotoxic damage to or kill the keratinocytes by induction of apoptosis.

Injury to the basal keratinocytes and other structures produce tiny vacuoles along the dermoepidermal junction on both sides of the basal lamina known as vacuolar alteration or vacuolization. ${ }^{2}$

Immunohistochemical studies of these reactions have demonstrated cytotoxic $\mathrm{T}$ cells ascending into the epidermis and presence of helper $\mathrm{T}$ cells in the papillary dermis. ${ }^{2}$ Immune complex deposition might also play a role in some interface dermatitis and antibody mediated cellular cytotoxicity could be the key in others. ${ }^{2}$ 
Le Boit P E, 1 has classified interface dermatitis into 5 types:

1. Interface Dermatitis with premature terminal differentiation.

2. Acute cytotoxic type.

3. Interface Dermatitis with psoariasiform hyperplasia.

4. Interface Dermatitis with irregular epidermal hyperplasia.

5. Interface dermatitis with epidermal atrophy.

The clinical lesions produced by an Interface Dermatitis may be flat or raised, smooth or scaly depending on the epidermal reaction.

Interface dermatitis encompasses a number of different clinical entities with diverse histological features. The rationale for grouping these conditions together under the term Interface Dermatitis is their histological similarity and presumably shared pathogenetic mechanism.

This study is aimed at understanding in detail the various associated histological features with clinical correlation of conditions in which Interface Dermatitis is the most important histopathologic finding. This will help us in arriving at a more specific diagnosis by light microscopy. It is also hoped that a better understanding of different conditions with shared pathogenesis will help in better patient care.

\section{AIMS OF THE STUDY:}

1. To study in detail histopathological findings associated with inferface dermatitis.

2. To classify the clinical conditions according to the type of interface dermatitis.

3. To correlate the clinical findings and diagnosis with the type of interface dermatitis.

MATERIALS AND METHODS: The study was conducted over a period of two and a half years. The skin biopsy specimens were fixed in $10 \%$ formalin and were processed entirely as per standard protocols. $4 \mathrm{~mm}$ thick sections were prepared and stained with Hematoxylin and Eosin. PAS staining was done in all cases.

All cases showing interface dermatitis as a major histological finding were included. A total of 71 cases were studied.

All sections were examined in detail and the associated histological features of each category of interface dermatitis were noted. Specific histological features aiding in differentiation of each category was assessed. PAS staining was done to highlight pathology of basement membrane. Normal skin biopsies \& biopsies with an unrelated condition were taken as control.

The cases were classified according to Le Boit's classification. Correlation between clinical features, clinical diagnosis and histological diagnosis was assessed.

RESULTS: During the period of two and a half years, 3015 skin biopsies were recieved for histopathological examination. The number of skin biopsy specimens featuring interface dermatitis as the major histopathological finding accounted for $2.35 \%$ of the total skin biopsies (71 cases).

The age of patients with interface dermatitis ranged from 8 years to 72 years with most of them being in the 50-59 year age group. (26.6\%) Majority of them were females (71.8\%). None of the patients had a family member with a similar illness. Five of the patients were diabetic and an equal number were hypertensive.

The most common clinical diagnosis offered was lichen planus (LP) followed by discoid lupus erythemathosus (DLE) as shown in Table 1. 


\section{Detailed Histopathological Examination:}

All cases showing Interface dermatitis were analysed with respect to specific histopathologic features.

1. Epidermal Changes: All cases of LP showed hyperkeratosis, hypergranulosis, irregular acanthosis \& basal cell vacuolation. (Figure 1) Colloid bodies were seen in 20 cases. (48.7\%). The case of Hypertrophic Lichen Planus (HTLP) showed hyperkeratosis, hypergranulosis, acanthosis and basal cell vacuolation.

Hyperkeratosis with atrophic epidermis and basal cell vacuolation was seen in all cases of DLE, Systemic lupus erythematosus (SLE) and Atrophic Lichen planus (ALP). (Figure 3 \& 4). Further, follicular plugging was present in 8 cases $(72.7 \%)$ and colloid bodies in 3 cases (27.2\%) of DLE. Atrophic epidermis and basal cell vacuolation was also seen in Dermatomyositis (DM). 2 of the cases of Bullous pemphigoid (BP) had hyperkeratotic and acanthotic epidermis and all cases showed spongiosis with basal cell vacuolation. All cases of Erythema multiforme (EM) showed spongiosis of epidermis with basal cell vacuolation.

2. Nature \& location of the dermal infilitrate: $35(85 \%)$ of the 41 cases of LP showed dense band like lymphoplasmacytic infiltrate in the dermo-epidermal junction while ALP cases showed only a minimal band like infiltrate.

Perivascular mononuclear inflammation was seen in all cases of EM, 8 cases of DLE, 3 cases of SLE and 2 cases of BP. Further, all cases of BP showed infiltration of epidermis and dermis by eosinophils.

Periappendageal inflammation was noted in 6 cases of DLE and 3 cases of SLE. All the cases of LP and HTLP had pigment incontinence which was also seen in 8 cases of DLE and 3 cases of SLE.

3. Histochemistry: PAS staining revealed focal areas of basement membrane disruption in all cases of LP, ALP, HTLP, EM, BP \& DM. 8(73\%) cases of DLE \& 1 (20\%) case of SLE also showed basement membrane disruption.(Figure 5) All cases of DLE \& SLE showed basement membrane thickening.(Figure 6)

\section{Application of Le Boit's classification:}

Type I or interface dermatitis with premature terminal differentiation accounted for majority $(57.7 \%)$ of the cases consisting of all cases of LP. Next in frequency was Interface dermatitis with epidermal atrophy (Type - 5) (29.1\%). Least common was interface dermatitis with irregular epidermal hyperplasia(1.4\%) as shown in Table -2 .

\section{Clinical presentation:}

a. Site distribution: $80 \%$ cases of LP had localized lesions confined to the flexor aspect of the extremities. 75\% cases of EM, DM and HTLP case had lesions confined to the extremities. All cases of SLE, DLE and 50\% cases of BP has generalized lesions with head and neck involvement. Oral lesions were seen in $20 \%$ cases of LP and nail involvement was seen in only 1 case of LP. 
b. Symptoms: Pruritus was the commomest symptom followed by photosensitivity and alopecia as seen in table 3.

c. Nature of lesions: Papules and plaques were the predominant lesions in LP as seen in table 4. Vesicles and bullae were seen only in EM and BP. Patients with DLE had predominantly plaque-like lesions.

\section{Percentage correlation between clinical diagnosis and histopathological diagnosis:}

$100 \%$ correlation was noted for DLE, SLE, HTLP \& DM. LP was diagnosed as lichenoid psoariasis in two cases and as porokeratosis in 1 case. 3 out of the 4 cases each of ALP, EM and BP correlated with clinical diagnosis.

DISCUSSION: One of the most challenging aspects in dermatopathology is to try to make specific diagnosis of inflammatory skin diseases. In this study, an attempt has been made at understanding in detail the various associated histological features with clinical correlation of conditions in which interface dermatitis is the most important histopathologic finding. This will help in arriving at a more specific diagnosis by light microscopy.

A total number of 71 cases of interface dermatitis were studied which showed significant female preponderance. Female preponderance has been noted for LP and SLE2,3. Majority of patients belonged to the 6 th decade(26.6\%). Boyd et $\mathrm{al}^{3}$ have reported a familial incidence of Lichen planus of $10.7 \%$. However, none of the patients had a family history in this study. 5 of the patients had associated diabetes mellitus \& 5 patients had associated hypertension. Jolly et al ${ }^{4}$ reported an incidence of $12.8 \%-85 \%$ of Lichen planus in diabetics.

Histopathology: All cases of LP showed thickening of the stratum corneum with orthokeratosis and hypergranulosis. The epidermis showed irregular acanthosis with saw tooth pattern. This conforms to findings reported by Boyd et $\mathrm{al}^{3}$. Max Joseph spaces (Figure 2) and Civatte bodies were noted in $12 \%$ and $48 \%$ of the cases respectively. A study by Ellis et al ${ }^{5}$ reported an incidence of $17 \%$ and $37-$ $100 \%$ respectively. All cases showed moderate to dense band like lymphoplasmacytic infiltrate in the papillary dermis with basal cell destruction similar to reports by Ellis et al ${ }^{5}$.

HTLP case showed hyperkeratosis and hypergranulosis with extensive irregular acanthosis of epidermis. Papillary dermis showed dense band like mononuclear inflammatory infiltrate with basal cell destruction. Cram et $\mathrm{al}^{6}$ reported pronounced vertically oriented fibrosis of the papillary dermis which was not seen in our case.

All cases of atrophic LP had hyperkeratosis with an atrophic epidermis and focal basal cell destruction. Papillary dermis showed minimal band like inflammatory infiltrate composed of lymphocytes and plasma cells. Black et $\mathrm{al}^{7}$ reported fibrosis in the papillary dermis consistent with the resolving stage of the disease which was not observed in our cases.

All cases of EM showed spongiosis of epidermis \& focal basal cell vacuolation. Papillary dermis showed perivascular lymphocytic infiltrate and 1 case showed subepidermal vesiculation. Le Boit et al has reported perivasular lymphocytes around the superficial plexus in early lesions and subepidermal vesiculation due to confluence of clefts at dermoepidermal junction. ${ }^{1}$

All cases of BP showed spongiosis of epidermis with focal basal cell vacuolation. Lower epidermis \& papillary dermis showed eosinophilic infiltration similar to study by Lever et al. ${ }^{8}$ 
All cases of SLE and DLE showed hyperkeratosis \& atrophy of epidermis. Focal vacuolar alteration of basal cell layer which constitutes an important diagnostic sign as described by Lever et $\mathrm{al}^{8}$ was seen in all cases of SLE and DLE. 3 cases of SLE and 8 cases of DLE showed follicular plugging, perivascular lymphocytic infiltrate in the papillary dermis and extravasation of RBCs. Le Boit et al ${ }^{1}$ reported that lymphocytic infiltrate in DLE is accompanied by fibrin thrombi within the vessel lumina, which was not seen in our cases.3 cases of SLE and 6 cases of DLE had periappendageal inflammatory infiltrate. Colloid bodies were seen in 3 cases of DLE. They have been reported by Lever et $\mathrm{al}^{8}$. Le Boit et $\mathrm{al}^{1}$ have reported that colloid bodies in DLE are evidence of damage to basal cell layer.

Lesions of DM showed hyperkeratosis with an atrophic epidermis and minimal periappendageal lymphocytic infiltrate in the dermis. Le Boit et $\mathrm{al}^{1}$ have reported that erythematous lesions of DM resemble early lesions of DLE. It is differentiated by the presence of extensive basal cell layer degeneration in DLE as against subtle changes in DM according to Lever8.

Histochemical Study: The use of PAS stain helped in highlighting the pathology of basement membrane. All cases of LP, HTLP, ALP, EM, BP, and DM showed focal fragmentation and destruction of basement membrane as compared to normal controls. Colloid bodies were seen to be PAS positive as reported by Lever et al. ${ }^{8}$

All the cases of DLE \& SLE showed thickening and tortuosity of basement membrane. Lever et al ${ }^{8}$ have reported that capillary walls may also show thickening, homogenisation and an increase in the intensity of the PAS reaction in DLE which was not evident in our study.

\section{Classification of cases according to the type of ID:}

The cases recieved in the study were classified based on the type of interface dermatitis. Majority belonged to interface dermatitis with premature terminal differentation or type I (57.7\%). The only condition representative of this type in this study was lichen planus and showed all the important histological findings reported in literature $1,3,8$. Next in frequency was Interface dermatitis with epidermal atrophy (29.9\%) with DLE being the most common condition(15\%). All the cases of DLE were of the atrophic type with perivascular and periappendageal inflammation. Le Boit ${ }^{1}$ has classified DLE with dense band like mononuclear infiltrate as ID Type I and atrophic lesions as Type 5. None of our cases of DLE showed features of Type I ID. The least common was Interface dermatitis with irregular epidermal hyperplasia represented by hypertrophic lichen planus(1.4\%). Altman $\mathrm{J}^{9}$ has reported an incidence of 6-19\%.

\section{Clinical correlation with the type of interface dermatitis:}

1. Interface dermatitis with premature terminal differentiation: $80 \%$ cases of LP had lesions localised to the flexor aspect of extremities, a finding similar to Boyd et al. ${ }^{3}$ Oral lesions were present in $16.9 \%$ cases while a higher incidence $(65 \%)$ was reported in classical cutaneous LP by Strauss etal. ${ }^{10}$ Arndt K. A ${ }^{11}$ reported genital involvement in $25 \%$ of men with typical skin lesions which was not seen in our study. Altman $\mathrm{J}^{9}$ reported nail involvement in $1-16 \%$ of patients. Our study had one case $(2.4 \%)$ with nail involvement.

Majority $(82.9 \%)$ of patients were symptomatic \& had intense pruritus. None of the patients had photosensitivity or alopecia. These findings are similar to those observed by Fellner MJ.12 
All patients had multiple lesions with most common lesions being papules \& plaques with a violaceous hue. Some lesions were erythematous. Similar findings have been reported by Boyd et al. ${ }^{3}$

2. Acute cytotoxic type: $75 \%$ of patients with EM had localised lesions with involvement of extremities and no mucosal or oral lesions. This conforms to reports by Le Boit PE. ${ }^{1}$ Two of the patients were symptomatic and had pruritus while none of them had photo sensitivity or alopecia. All patients presented with multiple erythematous papules \& vesicles. Le Boit PE1 have reported erythematous \& urticarial papules, papulo vesicles \& plaques.

3. Interface dermatitis with psoariasiform hyperplasia: All patients with BP had generalised lesions in the form of vesicles and bullae on an erythematous base. One case had oral lesions. Two patients had pruritic lesions while none had photo sensitivity or alopecia. Similar clinical findings have been reported by Lever et al. ${ }^{8}$

4. Interface dermatitis with irregular epidermal hyperplasia: The single case of HTLP representative of this type of ID in our study had localised involvement of the extensor aspect of lower limb in the form of multiple hyperpigmented plaques \& papules with no mucosal or nail involvement similar to reports by Boyd et al. ${ }^{3}$ The patient had no pruritus as against report of intensely pruritic lesions by Boyd et al. ${ }^{3}$

5. Interface dermatitis with epidermal atrophy: The most common condition belonging to this type of interface dermatitis in our study was DLE (15\%). Majority of the patients with DLE had generalised involvement of head \& neck and trunk in the form of multiple erythematous plaques with five of them showing central hypopigmentation. Oral lesions were present in a minority. Three of the patients had pruritic lesions, two of them had alopecia \& one had photo sensitivity. Thus photo sensitivity \& alopecia can be said to be associated with DLE. This conforms to reports by Lever et al. ${ }^{8}$

Five patients with SLE in this study had generalised lesions associated with photosensitivity. All patients had multiple erythematous papules \& plaques. One of the patients had scaling lesions. There have been similar findings described by Lever et al. ${ }^{8}$

All patients with atrophic LP in this study had generalised hypopigmented macules \& patches with no mucosal or nail involvement. There was no significant pruritus, photo sensitivity or alopecia. This conforms to reports by Boyd et al. ${ }^{3}$

Correlation between clinical diagnosis and histopathological diagnosis: Correlation between the clinical diagnosis and the histopathological diagnosis was 100\% for DLE, SLE, DM and HTLP. However, an alternative diagnoses was offered in $8.4 \%$ of the cases, thus emphasizes the role of histopathological examination in diagnosing dermatological conditions.

CONCLUSION: The study depicts the incidence, age and sex distribution of cases with interface dermatitis in our population. Type I interface dermatits with premature terminal differentiation was found to constitute the majority of cases followed by Type V. Histochemical studies highlight the basement membrane pathology. 
The study has shown that Interface Dermatitis occurs in a wide variety of clinicopathologic settings. A detailed light microscopic examination to evaluate subtle microscopic changes will help in arriving at a specific diagnosis. Direct immunoflourescence studies for immunoreactants in colloid bodies and dermoepidermal junction will further aid in establishing a diagnosis of interface dermatitis.

A better understanding of the different conditions with shared pathogenesis will help in better patient care.

The number of clinical conditions belonging to the different types of interface dermatitis in our study was limited. Hence, more studies are needed in order to identify specific features to redefine each of the entities.

\section{REFERENCES:}

1. Le Boit PE Interface dermatitis. How specific are its histopathologic features? Arch. Dermatol 1993 Oct. 129: 1324-8.

2. Maize et al. Cutaneous pathology. Churchill Livingstone 1998, p - 85-145.

3. Boyd A. S, Neldner K H: Lichen Planus J Am Acad Dermatol 1991 25: 593-619.

4. Jolly M. Lichen planus and its association with Diabetes Mellitus. Med J Aust 1972 1: 990 -2

5. Ellis FA. Histopathology of lichen planus based on analysis of one hundred biopsy specimens. J Invest dermatol 1967, 48: 143-8.

6. Cram DL, Muller SA. Unusual variations of lichen planus Mayo Clin Proc 1966; 41: 677-88.

7. Black M M. What is going on in Lichen planus? Clin Exper Dermatol 1977:2,303.

8. Lever's Histopathology of the Skin. Eighth edition. Lippincott 1997, p-166-261.

9. Altman J, Perry H 0. The variations and course of Lichen Planus. Arch. Dermatol 1961, 84: 17991 (84: 179-91).

10. Strauss R A, Fattore L, Soltani K: The association of mucocutaneous lichen planus and chronic liver disease.Oral Pathol 1989, 68: 406-10.

11. Arndt KA. In: Fitz patrick TB, Eisen AZ, Wolff K etal eds. Dermatology in General Medicine $3^{\text {rd }}$ ed. New York MC Graw Hill 1987, 967-73.

12. Fellner M J. Lichen Planus. Int J Dermatol 1980, 19: 71-5.

\begin{tabular}{|c|c|c|}
\hline Condition & Number & $\%$ \\
\hline LP & 41 & $57.7 \%$ \\
\hline EM & 4 & $5.6 \%$ \\
\hline $\mathrm{BP}$ & 4 & $5.6 \%$ \\
\hline HTLP & 1 & $1.4 \%$ \\
\hline DLE & 11 & $15.4 \%$ \\
\hline SLE & 5 & $7 \%$ \\
\hline ALP & 4 & $5.6 \%$ \\
\hline $\mathrm{DM}$ & 1 & $1.4 \%$ \\
\hline Total & 71 & $100 \%$ \\
\hline
\end{tabular}


ORIGINAL ARTICLE

\begin{tabular}{|c|c|c|c|}
\hline Type of ID & Clinical condition & No. & $\mathbf{\%}$ \\
\hline I & LP & 41 & $57.7 \%$ \\
\hline II & EM & 4 & $5.6 \%$ \\
\hline III & BP & 4 & $5.6 \%$ \\
\hline IV & HTLP & 1 & $1.4 \%$ \\
\hline V & DLE & 11 & $15 \%$ \\
\hline & SLE & 5 & $7 \%$ \\
\cline { 2 - 4 } & ALP & 4 & $5.6 \%$ \\
\cline { 2 - 4 } & DM & 1 & $1.4 \%$ \\
\hline \multirow{2}{*}{ Total } & & $\mathbf{7 1}$ & $\mathbf{1 0 0} \%$ \\
\hline
\end{tabular}

Table 2: Frequency of different types of interface dermatitis

\begin{tabular}{|c|c|c|c|c|c|c|c|c|c|}
\hline Symptoms & LP(41) & ALP(4) & HTLP(1) & DLE(11) & SLE(5) & EM(4) & BP(4) & DM(1) & Total(71) \\
\hline Pruritus & $34(82.9)$ & $1(25)$ & - & $3(27.2)$ & $1(20)$ & $2(50)$ & $2(50)$ & - & $43(60.5)$ \\
\hline $\begin{array}{c}\text { Photo } \\
\text { sensitivity }\end{array}$ & - & - & - & $1(9)$ & $3(60)$ & - & - & - & $4(5.6)$ \\
\hline Alopecia & - & - & - & $2(18.1)$ & - & - & - & - & $2(2.8)$ \\
\hline \multicolumn{7}{|c|}{ Table 3: Incidence of clinical features }
\end{tabular}

\begin{tabular}{|c|c|c|c|c|c|c|c|c|c|}
\hline $\begin{array}{c}\text { Nature of } \\
\text { lesions }\end{array}$ & LP (41) & ALP(4) & HTLP(1) & DLE(11) & SLE(5) & EM(4) & BP (4) & DM(1) & Total71 \\
\hline Macules & $9(21.9)$ & $3(75)$ & - & $2(18.1)$ & - & - & - & - & $14(19.7)$ \\
\hline Papules & $21(51.2)$ & - & - & $2(18.1)$ & $1(20)$ & $2(50)$ & - & $1(100)$ & $27(38)$ \\
\hline Plaques & $29(70.7)$ & - & $1(100)$ & $5(12.1)$ & $3(60)$ & - & - & - & $38(53.5)$ \\
\hline Patches & $6(14.6)$ & $2(50)$ & - & $1(9)$ & $1(20)$ & - & - & - & $10(14)$ \\
\hline Vesicles & - & - & - & - & - & $4(100)$ & $2(50)$ & - & $6(8.4)$ \\
\hline Bullae & - & - & - & - & - & $4(100)$ & $4(100)$ & - & $8(11.2)$ \\
\hline
\end{tabular}

\section{Table 4: Nature of lesions}

Fig. 1: Lichen planus- hyperkeratosis, hypergranulosis, irregular acanthosis \& dense mononuclear infiltrate in papillary dermis. (H \& E, 100X) Inset: basal cell degeneration (H \& E, 400X).

Fig. 2: Lichen planus- Max Joseph space (H\&E, 100X).

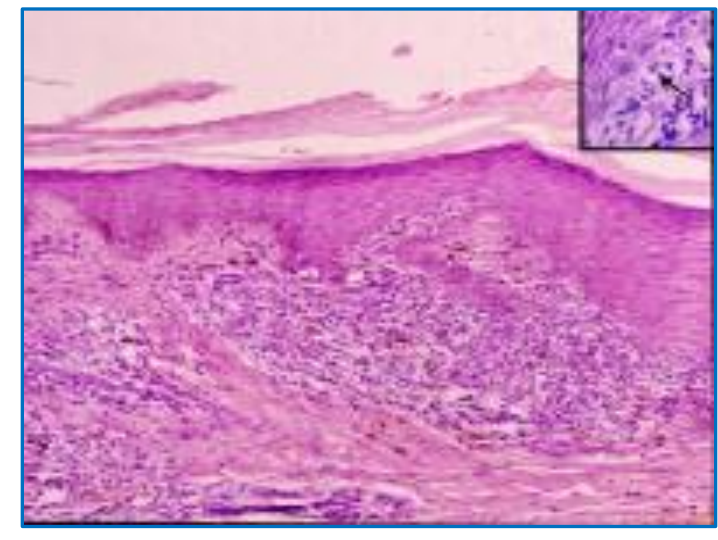

Fig. 1

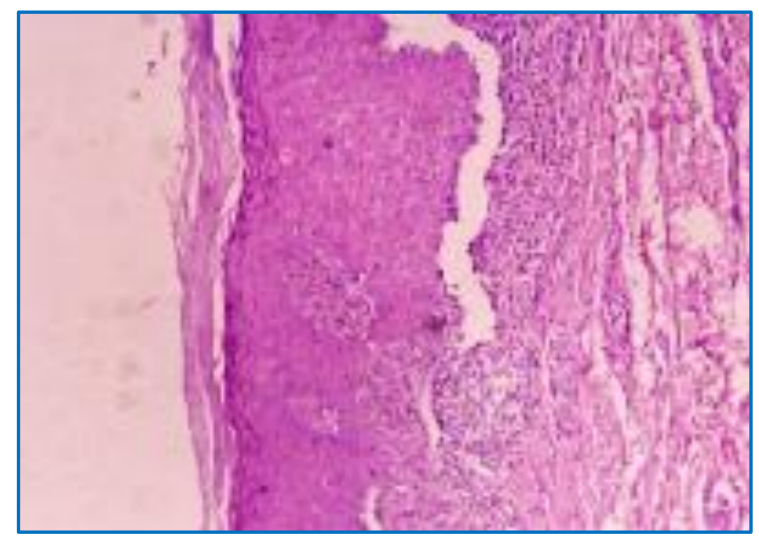

Fig. 2 


\section{ORIGINAL ARTICLE}

Fig. 3: Discoid lupus erythematosus- atrophic epidermis with follicular plugging (H\&E, 100X).

Fig. 4: Systemic lupus erythematosus- atrophic epidermis with basal cell vacuolation (H\&E, 200X).

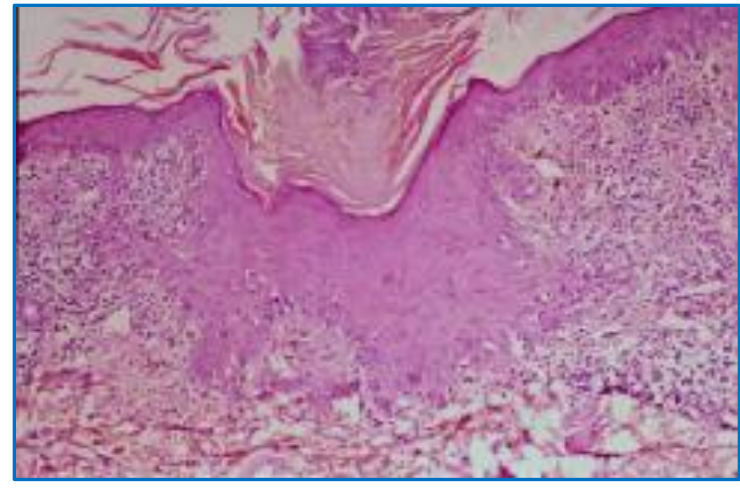

Fig. 3

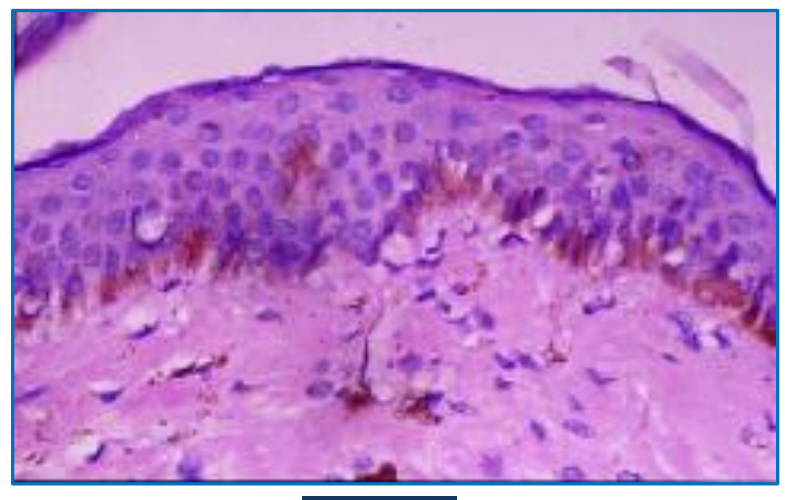

Fig. 4

Fig. 5: Lichen planus- basement membrane disruption (PAS, 100X).

Fig. 6: Discoid lupus erythematosus- basement membrane thickening (PAS, 100X).

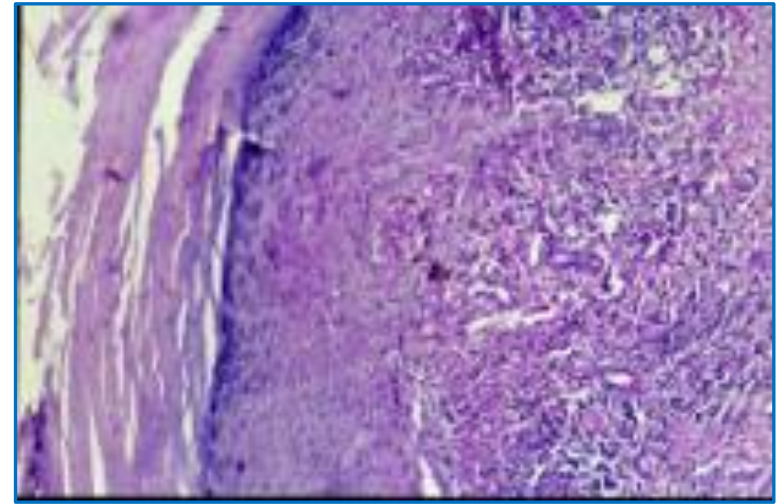

Fig. 5

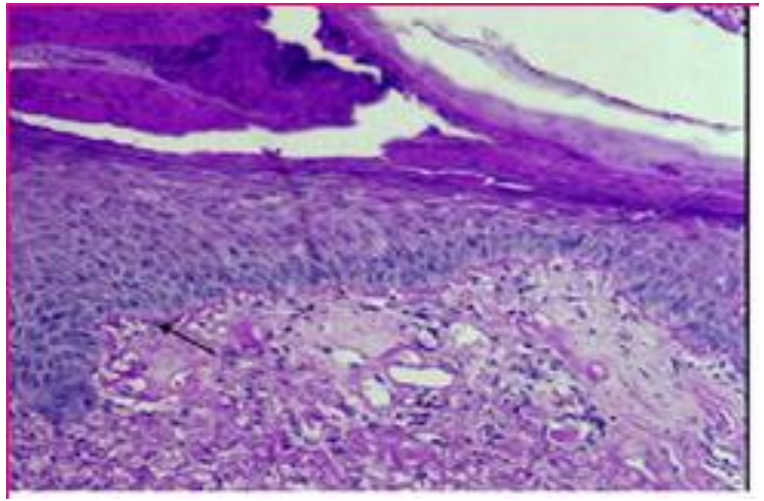

Fig. 6

\section{AUTHORS:}

1. Suja Ajoy Kumar

2. G. Nandakumar

\section{PARTICULARS OF CONTRIBUTORS:}

1. Associate Professor, Department of Pathology, Kempegowda Institute of Medical Sciences, Bangalore.

2. Additional Professor, Department of Pathology, Government Medical College, Trivandrum, Kerala.

FINANCIAL OR OTHER COMPETING INTERESTS: None

\section{NAME ADDRESS EMAIL ID OF THE CORRESPONDING AUTHOR:}

Dr. Suja Ajoy Kumar, N 501, Purva Highlands, Mallasandra, Off Kanakpura Road, Bangalore-560062.

E-mail: sujaajoy@yahoo.co.in

Date of Submission: 30/04/2015.

Date of Peer Review: 01/05/2015.

Date of Acceptance: 19/05/2015.

Date of Publishing: 23/05/2015. 